\title{
Some relationships between radiative and atmospheric quantities through 1D NLTE modeling of prominences in the Mg II lines
}

\author{
J.-C. Vial, P. Zhang, and É. Buchlin
}

\author{
Institut d'Astrophysique Spatiale, CNRS/Université Paris-Sud, Université Paris-Saclay, Bâtiment 121, Université Paris-Sud, \\ 91405 Orsay Cedex, France \\ e-mail: jean-claude.vial@ias.u-psud.fr
}

Received 14 September 2018 / Accepted 19 February 2019

\begin{abstract}
Context. With more than four years of IRIS observations, and in order to avoid building customized diagnostics for each observation, it is useful to derive some simple relations between spectra and physical quantities. This is even more useful for the $\mathrm{k}$ and $\mathrm{h}$ lines of $\mathrm{Mg}$ II, which require complex non-local-thermodynamic-equilibrium NLTE treatments.

Aims. The aim of this work concerning prominences is to correlate observable spectral features in $\mathrm{h}$ and $\mathrm{k}$ lines of Mg II to physical quantities such as the density and the emission measure (EM) in the same way as similar correlations have been obtained in the hydrogen lines. In this way, and within approximations done on some parameters such as temperature, it is possible to build pixel by pixel an IRIS map of the above-mentioned quantities.

Methods. In order to simplify and shorten the modeling, we chose to compute one-dimensional (1D) isothermal and isobaric models that are treated with the PROM7 NLTE code available at MEDOC (IAS). We built a set of models with large ranges of temperature, pressure, and thickness. At all altitudes considered, we paid attention to the exact computation of the incident radiation. Then we compared the emergent $\mathrm{Mg}$ II $\mathrm{h}$ and $\mathrm{k}$ intensities with the corresponding hydrogen and electron densities and EMs.

Results. From the NLTE computation, we derive correlations between the $\mathrm{k}$ and $\mathrm{h}$ emergent intensities on one hand and the densities and EM on the other hand. With some assumptions on the temperature, we obtain a unique relation between the $\mathrm{k}$ (and $\mathrm{h}$ ) intensities and the EM that should be useful for deriving either the hydrogen and electron densities or the effective thickness of an observed prominence.

Conclusions. From NLTE modeling, we have provided a relationship between observable integrated intensities of the $\mathrm{Mg}_{\text {II }}$ resonance lines and prominence plasma EM, which will contribute to a first-order analysis of long time series of spectroscopic observations, for example, with IRIS. We anticipate building more complex relations between the profiles and other plasma quantities.
\end{abstract}

Key words. line: formation - radiative transfer - Sun: filaments, prominences - Sun: UV radiation

\section{Introduction}

The thermodynamic conditions in solar prominences (Vial \& Engvold 2015) are rather close to the conditions met in the solar chromosphere, from the low chromosphere to the transition region and up to the corona. The temperature ranges from about $6000 \mathrm{~K}$ to a few $100000 \mathrm{~K}$ (in the transition region to the corona) and the density, although smaller than in the low chromosphere, is a few $10^{11} \mathrm{~cm}^{-3}$ (Parenti 2015; Labrosse 2015). As in the chromosphere, the plasma is partially ionized and the plasma $\beta$ is smaller than unity. The plasma is also in non-local-thermodynamic-equilibrium (NLTE) conditions, conditions "aggravated" by the fact that solar prominences are illuminated by strong ionizing and exciting radiations from the chromosphere and the corona. The diagnostic of such structures requires specific NLTE codes, which were first built in onedimension geometry (1D; see the pioneering work of Heasley \& Mihalas 1976; Heasley \& Milkey 1976, 1983). These 1D codes proved to be very useful for deriving density, temperature, and other quantities (such as the ionization degree). A systematic modeling, as performed by Gouttebroze et al. (1993; hereafter GHV) with 140 models, not only provided specific signatures but also allowed for the derivation of relations between model parameters and emitted lines and continua (Heinzel et al. 1994; hereafter HGV). As an example, it was shown (Fig. 15 of HGV) that the $\mathrm{H} \alpha$ intensity is proportional to the emission measure (EM, square of the electron density integrated on the line of sight), at least for temperatures lower than $15000 \mathrm{~K}$.

Over the last thirty years, this modeling has not been limited to hydrogen but has also been extended to other elements such as He I, He II, Ca II, Na I, and Mg II (Labrosse et al. 2010; Labrosse 2015). Hydrogen (and helium) modeling is always a necessary step in order to properly treat the ionization and to assign the electron density. Of course, in view of the amazing complexity of the geometry of prominences, it was felt necessary to build and use 2D codes (e.g., Vial 1982; Paletou et al. 1993; Heinzel \& Anzer 2001) to better describe the boundaries of the structures. Other improvements have been made through grafting a transition region describing the so-called prominence corona transition region (PCTR) onto an otherwise isobaric and isothermal layer (see, e.g., Heinzel 2015). Another major step was performed with the introduction of "thread models" where a layer is replaced by the addition of numerous much smaller onedimensional layers (Gunár et al. 2007). Based upon magnetohydrostatic equilibrium, 2D vertical-thread models have been built and their spectral signatures (e.g., in the Lyman lines of hydrogen) compared in two extreme situations: a line of sight (LOS) parallel and perpendicular to the thread (Heinzel et al. 2005). Such 
models allow for the comparison with high resolution observations where the geometry of the prominence is (more or less) well determined, and particularly the general orientation of the threads (i.e., the field lines) with respect to the line of sight (LOS) in terms of local PCTR and velocity fields. Such observations are very rare and hampered, as all limb observations are, by LOS effects such as multi-structures or precise orientation.

One can be tempted to search within a very large set of $2 \mathrm{D}$ models with PCTR for the best one that fits with an observed profile and then to derive the local properties. Such an approach will certainly become more and more attractive with the advent of more complete observations (including stereoscopy) and the availability of more computing power. However, parameters obtained from such models, which depend on many parameters, are prone to overfitting, and the correct parameter values should not contradict, at first order, the basic 1D modeling. Moreover, a thorough study of large structures over a long time span (e.g., in the case of an eruptive prominence) requires the use of simple and robust tools such as 1D modeling.

In some important cases, the evaluation of the total density, even averaged, is of major importance: for instance, since prominences are maintained in the corona through a balance between gravity and the tension component of the magnetic pressure, the determination of the total mass is critical for selecting the right models that lead to destabilization and eruption. Also the determination of the mass flows, at the smallest scale allowed by the spatial resolution, requires a determination of the density at each pixel of the prominence image and at all times, a task made easier and faster with 1D models. However, because of the instrumental limitations, it may be necessary to build some space-averaged values of the densities (and velocities).

With the advent of the Interface Region Imaging Spectrograph (IRIS) mission (De Pontieu et al. 2014), it has been possible to observe prominences with the highest spatial and time resolutions in the $\mathrm{h}$ and $\mathrm{k}$ lines of $\mathrm{Mg}$ II. A lot of results have been obtained in both $\mathrm{Mg}$ II $\mathrm{h}$ and $\mathrm{k}$ observational and modeling domains (e.g., Heinzel et al. 2014; Vial et al. 2016). It is our objective here to derive some "laws" relating thermodynamic quantities to specific $\mathrm{Mg}$ II $\mathrm{h}$ and $\mathrm{k}$ signatures, in the same way as GHV and HGV did with hydrogen. The paper is organized as follows: in Sect. 2, we recall the main characteristics of our NLTE modeling with emphasis on the exact computation of the incident radiation. In Sect. 3, we present our main results in terms of correlations between the $\mathrm{k}$ and $\mathrm{h}$ integrated intensities with respect to main quantities (densities and EM). In our conclusion (Sect. 4), we emphasize the importance of the unique relation between the $\mathrm{k}$ intensity and the EM, of great interest for the derivation of density and consequently mass flows.

\section{One-dimensional NLTE modeling}

We use the NLTE radiative-transfer code PROM7 $7^{1}$ to compute the $\mathrm{Mg}$ II $\mathrm{h}$ and $\mathrm{k}$ synthetic spectra. The NLTE modeling is done in two steps. First, we compute the statistical equilibrium for a five-level plus continuum hydrogen atom to determine the electron densities at all depths; here we neglect the helium ionization. The resulting atmospheric structure is then used to solve the NLTE problem for magnesium. We used relatively simple 1D models in this study and concentrated on details of the $\mathrm{Mg}$ II line formation in a multilevel atom (5 levels and continuum), using the angle-averaged partial frequency redistribution

\footnotetext{
1 This code is available at https://idoc.ias.u-psud.fr/MEDOC/ Radiative\%20transfer\%20codes/PROM7
}

Table 1. Grid of 1D-slab models used in this work.

\begin{tabular}{ll}
\hline \hline Parameter & Values or range \\
\hline$T(\mathrm{~K})$ & $8000-14000$ \\
$p\left(\mathrm{dyn} \mathrm{cm}^{-2}\right)$ & $0.005-0.5$ \\
$D(\mathrm{~km})$ & $200,1000,5000$ \\
$H(\mathrm{~km})$ & 10000 \\
$V\left(\mathrm{~km} \mathrm{~s}^{-1}\right)$ & 5 \\
\hline
\end{tabular}

(PRD) hypothesis. However, let us mention that one could add a PCTR, but this corresponds to more parameters to control in a region where the $\mathrm{Mg}$ II ions only exist below about $15000 \mathrm{~K}$ (and certainly not above $20000 \mathrm{~K}$ ). Consequently, the prominence structure in this model consists of one-dimensional (1D) isothermal and isobaric plasma slabs vertically standing on the solar surface and irradiated by photospheric and chromospheric radiation.

The input parameters of this code include gas pressure, temperature, the height above the solar surface, geometrical thickness of the 1D slab, and microturbulent velocity. The outputs are several physical quantities and optical parameters like the electron density, the total hydrogen density, $\mathrm{Mg}_{\mathrm{I}}$, II and III ground state densities, the spectral profiles and integrated intensities of $\mathrm{H}$ lines and $\mathrm{Mg}$ lines, and their optical thickness.

With the results of the models, we want to investigate how a realistic range of prominence physical parameters will influence the properties of $\mathrm{Mg}$ II lines. We explore a grid of models and obtain a range of predicted line intensities that can be compared with the observed IRIS profiles (Zhang et al. 2019; hereafter Paper I).

\subsection{Models}

We constructed a grid of 3780 NLTE models, covering a temperature range of $8000-14000 \mathrm{~K}$, a pressure range of $0.05-$ $0.5 \mathrm{dyn} \mathrm{cm}^{-2}$, a height range of $10000-50000 \mathrm{~km}$, while the thickness varies between 200 and $5000 \mathrm{~km}$ and the turbulent velocity is $5 \mathrm{~km} \mathrm{~s}^{-1}$ for all the models. In Table 1 , we give the values of the prominence parameters for the models.

\subsection{Boundary conditions}

In the case of solar prominence modeling, the incident radiation intensity is a key boundary condition (Heinzel 2015). It is calculated by integrating the radiation received at a point at an arbitrary height above the solar surface in the corona over all of the atmosphere visible from this point (Brown \& Labrosse 2018), as seen in Fig. 5 of Sahal-Brechot et al. (1986). We compute the mean local specific intensity (in erg s${ }^{-1} \mathrm{sr}^{-1} \mathrm{~cm}^{-2} \AA^{-1}$ ) and we are able to take into account the effect of the geometry, the active regions on the solar surface, and the radial variation of the solar brightness.

The chromosphere being optically thick for Mg II k\&h lines, we simply assume that the incoming radiation mainly comes from the solar surface. We set the surface to be in the upper chromosphere, $3800 \mathrm{~km}$ above photosphere, which is the position of the Mg II limb, based on the analysis of the IRIS mosaic observation on May 27, 2014 (Paper I). The prominence is illuminated from all directions in the solar surface, and this radiation will be different for different directions. The mean intensity coming from all directions is derived by averaging over the solid 
angle $\Omega$, which limits the solar horizon of the point $\mathrm{M}$ in the prominence, at some height above the surface:

$J=\frac{1}{4 \pi} \oint_{\Omega} I(\Omega) \mathrm{d} \Omega$.

The contribution from the surface in different directions is computed from the radiation $I_{\mathrm{P}}$ at different points $\mathrm{P}$ on the solar surface by

$I_{\alpha}=\frac{1}{4 \pi} I_{\mathrm{P}} \Delta \Omega$

in which

$\Delta \Omega=\Delta S \cos \delta / \rho^{2}=\frac{\Delta S}{\rho^{2}} \sqrt{1-\left(1+\frac{h}{R_{\odot}}\right)^{2} \sin ^{2} \alpha}$,

where $\alpha$ is the angle between MP and the local vertical, $\rho=\mathrm{MP}$, $h=\mathrm{P}_{0} \mathrm{M}$ is the height of $\mathrm{M}$ above the limb, $R_{\odot}$ is the solar radius, and $\delta$ is the angle between the MP direction and the normal to the surface of the Sun.

In our computation, $J_{\mathrm{P}}$ is mainly derived from the absolute intensities of IRIS mosaic observations in $\mathrm{Mg}$ II $\mathrm{k} \& \mathrm{~h}$ lines. Once a month, mosaic observations in six spectral windows surrounding the strongest spectral lines ( $\mathrm{Mg}_{\text {II }} \mathrm{h}$ and $\mathrm{k}, \mathrm{C}$ II 1335 and $1334 \AA$, and Si IV 1393 and $1403 \AA$ ) are built from a series of rasters taken at different pointing locations that cover the full disk.

We also take into account the center-to-limb variation of the radiation at different heights above the solar surface. This variation is calculated from the IRIS mosaic observation taken on March 12, 2017, when there is no apparent active region on disk. We take it as a representation of the "mean quiet Sun" and derive the radial intensity variation for the $\mathrm{Mg}$ II $\mathrm{k} \& \mathrm{~h}$ spectrum. Figure 1 shows the derived center-to-limb variation at the wavelength of $k_{3}$, where $k_{1}, k_{2}$, and $k_{3}$ have their usual meaning. The variation exhibits limb darkening, and this effect may be caused by the variation of the formation height of $k_{3}$, while more highlying and dark fibrils are sampled close to the limb (Schmit et al. 2015). We also have limb darkening at the positions of the blue and red peaks of the $\mathrm{k} \& \mathrm{~h}$ lines, and changes from limb darkening to limb brightening in the wavelength range between $k_{2}$ and $k_{1}$. Let us state here that the $k_{2 v}$ position is defined as the position of the violet peak of the average (sun center) profile. It does not take into account peak-to-peak broadening towards the limb. This change may only be apparent and may be caused by the variation of the thermal broadening of the k\&h lines, which makes the peaks go further away from the line center from center to limb.

For our modeling purpose, we rely on the IRIS mosaic observation taken from 10:06 UT on May 27, 2014 to 3:30 UT on May 28, 2014 to derive the incident radiation coming from the surrounding solar atmosphere. As shown in Fig. 15 of Paper I, this observation is transformed into Carrington coordinates, and complemented by He II $304 \AA$ data from the Solar Terrestrial Relations Observatory STEREO A taken at 15:00 UT on May 28. In this figure, the regions inside the circles indicate where the incident radiation comes from at different heights over the surface. Clearly, since there is no active region inside the "horizon" as seen from the prominence, the incident radiation comes from quiet regions. This means that our modeling is valid for quiescent prominences only. Figure 2 shows the derived incident radiation profiles of $\mathrm{Mg}$ II $\mathrm{k} \& \mathrm{~h}$ lines at a height of $10000 \mathrm{~km}$ over the limb.
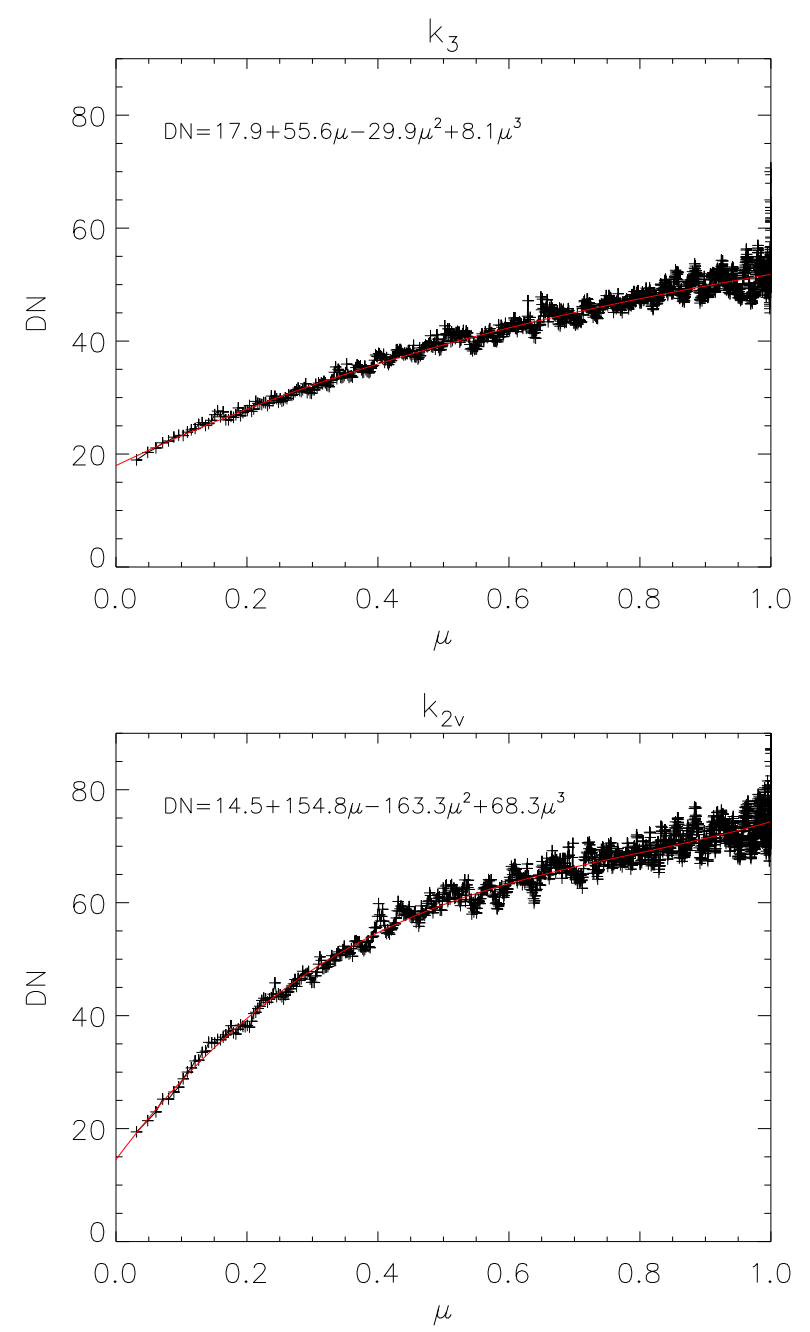

Fig. 1. Radial variation of intensity in $k_{3}$ and $k_{2 v}$. A polynomial fit as a function of $\mu$ is over-plotted in red.

\section{Results}

Our objective is to find radiative signatures (focused here on the $\mathrm{h}$ and $\mathrm{k}$ lines of $\mathrm{Mg}$ II) of some characteristic models. Since we have a multi-parameter space characterizing the model, we focus on the (integrated) $\mathrm{k}$ and $\mathrm{h}$ intensities as the radiative output of the modeling and the (total) hydrogen and electron densities as the main physical quantities of the model. The justification is as follows: firstly, we know that the $\mathrm{Mg}$ II lines are formed in a (relatively) narrow range of temperatures, essentially determined by the first two levels of ionization of $\mathrm{Mg}$ II (Heinzel et al. 2014). Actually, our modeling shows that the $\mathrm{Mg}$ II and $\mathrm{Mg}$ III populations become equal at a temperature less than $15000 \mathrm{~K}$, and the $\mathrm{Mg}$ III/ $\mathrm{Mg}$ II ratio is 100 at $20000 \mathrm{~K}$. Secondly, we want to check if the above-mentioned relationship between the $\mathrm{H} \alpha$ intensity and the EM could also be valid for the $\mathrm{h}$ and $\mathrm{k}$ lines. If so, it will offer a simple and supplementary tool for the diagnostic (density combined to thickness) of prominences. Let us note that a different approach has been taken by Jejčič et al. (2018) who focused on the reversal of the $h$ and $k$ profiles. We simultaneously varied the temperature (between 8000 and $14000 \mathrm{~K}$ ) and the thickness $(500,1000$ and $2500 \mathrm{~km})$ while the whole range of pressures (and consequently electron density) was explored. The microturbulence and height were fixed at $5 \mathrm{~km} \mathrm{~s}^{-1}$ and $10000 \mathrm{~km}$ respectively. As a first step, the radial velocity was set to $0 \mathrm{~km} \mathrm{~s}^{-1}$. 

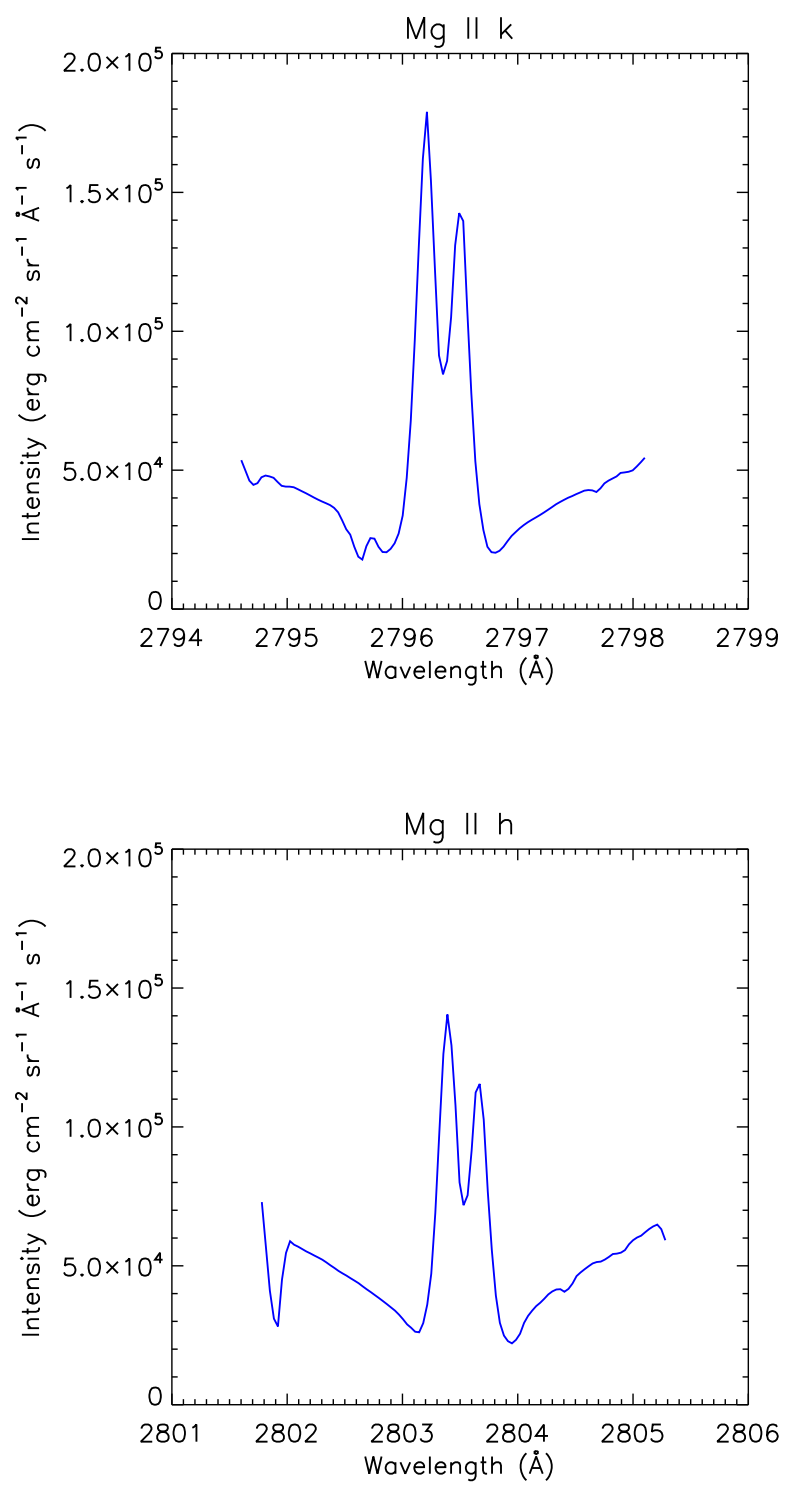

Fig. 2. Incident $\mathrm{Mg}$ II $\mathrm{h}$ and $\mathrm{k}$ profiles at an altitude of $10000 \mathrm{~km}$.

The EM was computed as the integral of the square of the density over the depth in the prominence. It was computed for the electron density but also for the (total) hydrogen density, which is the result of the hydrogen NLTE computations that are preliminary to the $\mathrm{Mg}$ modeling, for every single model. The $\mathrm{H}$ density is important since it contributes to the bulk of the total mass. It is used in the computation of mass flows derived from IRIS observations in Paper I. The results for both EM are shown on Fig. 3 for $\mathrm{Mg}$ II $\mathrm{k}$ and Fig. 4 for $\mathrm{Mg}$ II $\mathrm{h}$.

It is clear that we have a monotonous variation of $I_{\mathrm{k}}$ and $I_{\mathrm{h}}$ (the $\mathrm{Mg}$ II $\mathrm{k}$ and h emerging intensities) versus the two EMs when temperature and thickness are fixed. Moreover, the behavior of $I_{\mathrm{k}}$ and $I_{\mathrm{h}}$ is quite similar, a result that leads us to discuss $I_{\mathrm{k}}$ only for now. However, as shown by Alissandrakis et al. (2018), the addition of $I_{\mathrm{k}}$ and $I_{\mathrm{h}}$ information (or their ratio) can be used to further discriminate between models. We also remark that at high intensities $\left(I_{\mathrm{k}}>4 \times 10^{4} \mathrm{erg} \mathrm{cm}^{-2} \mathrm{sr}^{-1} \mathrm{~s}^{-1}\right)$, the curves are well separated with temperature for the hydrogen EM. This is not the case for the electron EM. Another interesting feature is that around $10000-11000 \mathrm{~K}$, for intensities lower than $2 \times 10^{4} \mathrm{erg} \mathrm{cm}^{-2} \mathrm{sr}^{-1} \mathrm{~s}^{-1}$, the curves seem not to depend on the thickness.
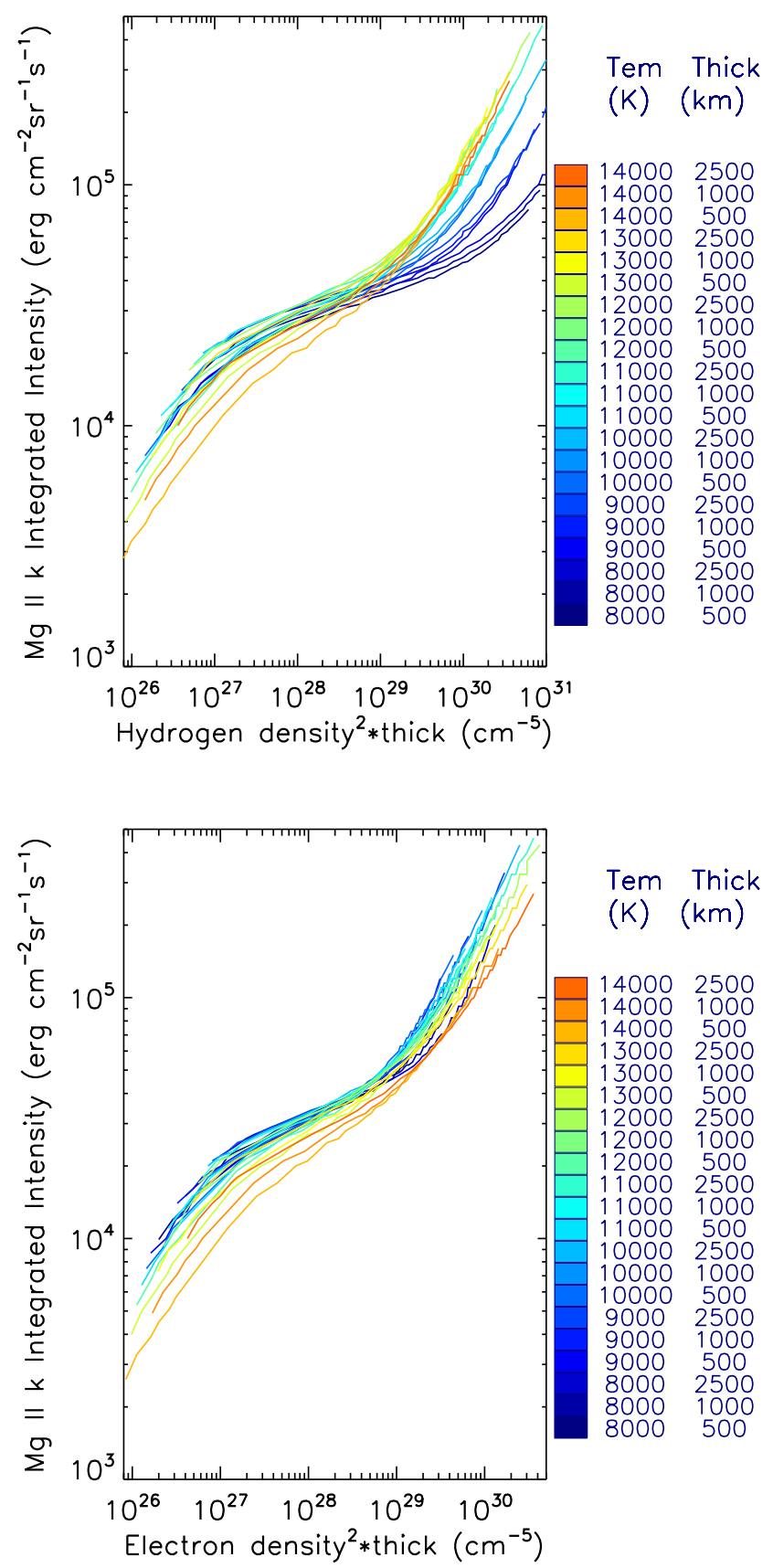

Fig. 3. Integrated $\mathrm{Mg}$ II $\mathrm{k}$ line intensity as a function of the hydrogen EM (top panel) and the electron EM (bottom panel).

Since the NLTE computations show the importance of the scattering of the incident radiation (e.g., Heinzel et al. 2014), its variation due to the Doppler effect of a radial velocity must be investigated. Figure 5 shows the effect of a modest radial velocity $\left(20 \mathrm{~km} \mathrm{~s}^{-1}\right)$, which leads to a slight increase of $I_{\mathrm{k}}$. One can note that for a given $I_{\mathrm{k}}$ intensity, the EM decreases by at most a factor of two for a velocity of $20 \mathrm{~km} \mathrm{~s}^{-1}$. Actually the effect of radial velocity (at $20 \mathrm{~km} \mathrm{~s}^{-1}$ ) disappears for electron EM above $10^{29} \mathrm{~cm}^{-5}$, because the scattering occurs inside a very opaque plasma, which leads to a completely incoherent frequency redistribution. We note that subtle coherent effects certainly occur in the wings with increasing radial velocity (see Gontikakis et al. 1997).

The variation of $I_{\mathrm{k}}$ with the hydrogen and electron EMs is well evidenced when one keeps the thickness constant and allows the temperature to vary (Fig. 6). The curves do not show 

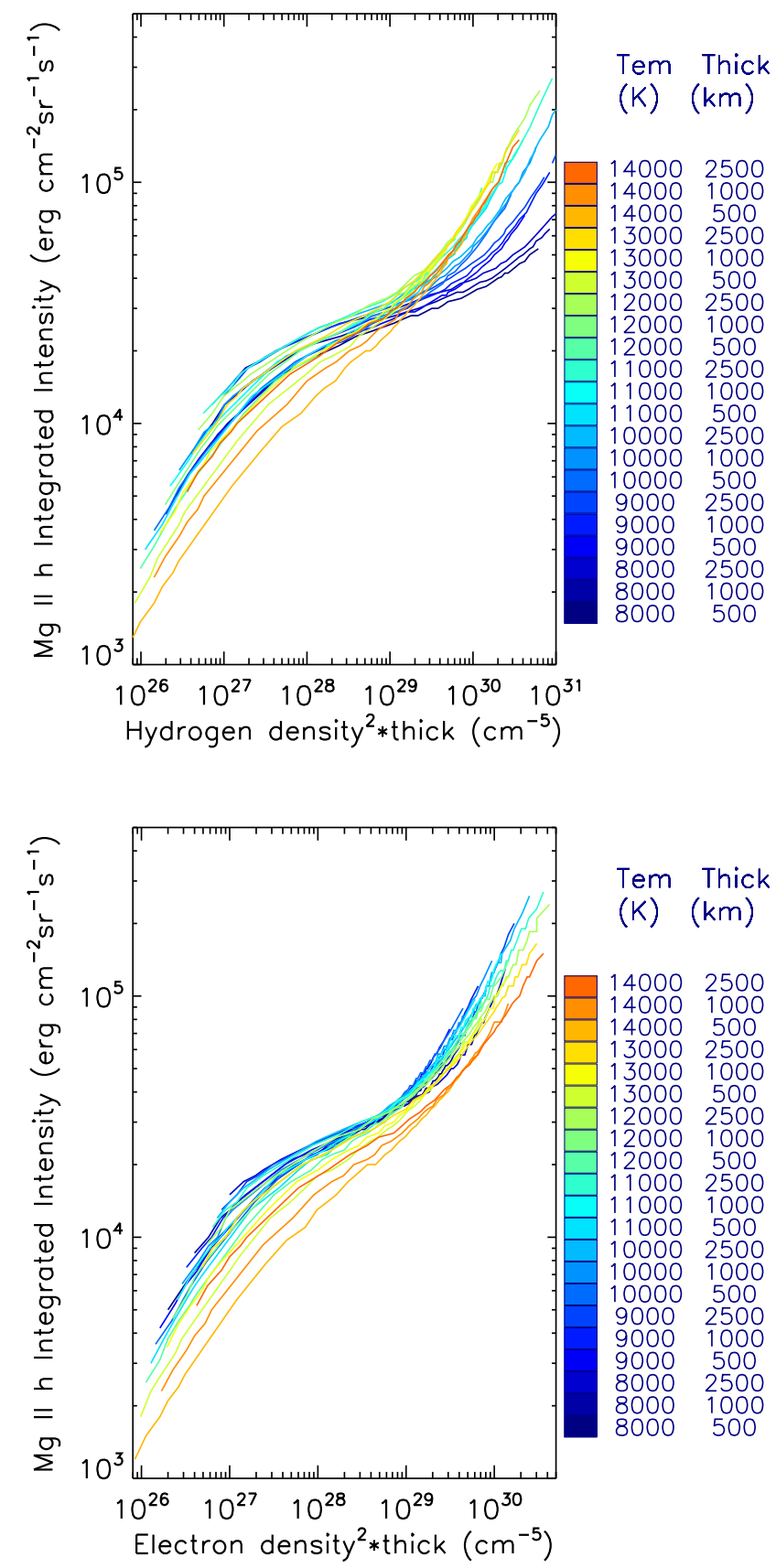

Fig. 4. Integrated $\mathrm{Mg}$ II $\mathrm{h}$ line intensity as a function of the hydrogen EM (top panel) and the electron EM (bottom panel).

strong variations with temperature for $\mathrm{k}$ intensity lower than $2 \times 10^{4}$, for temperatures lower than $13000 \mathrm{~K}$, and EM lower than about $2 \times 10^{29} \mathrm{~cm}^{-5}$.

Since some observations (Paper I) limit the ranges of $I_{\mathrm{k}}$ and temperatures values (Fig. 6), we take a step further and set the temperature to a fixed value of $11500 \mathrm{~K}$. The curves shown in the top panel of Fig. 7 exhibit a strong sensitivity to the value of the thickness, which is varied between 200 and $5000 \mathrm{~km}$. This plot provides the observer with a very useful tool for computing the average quadratic electron and hydrogen densities, since a high resolution instrument such as IRIS can separate threads of about $200 \mathrm{~km}$ : assuming a circular cross-section, one can derive the actual thickness of the threads (if they are well isolated). As far as the hydrogen EM is concerned, at the assumed temperature the curves lead to a unique relation linking $I_{\mathrm{k}}$ on one hand and
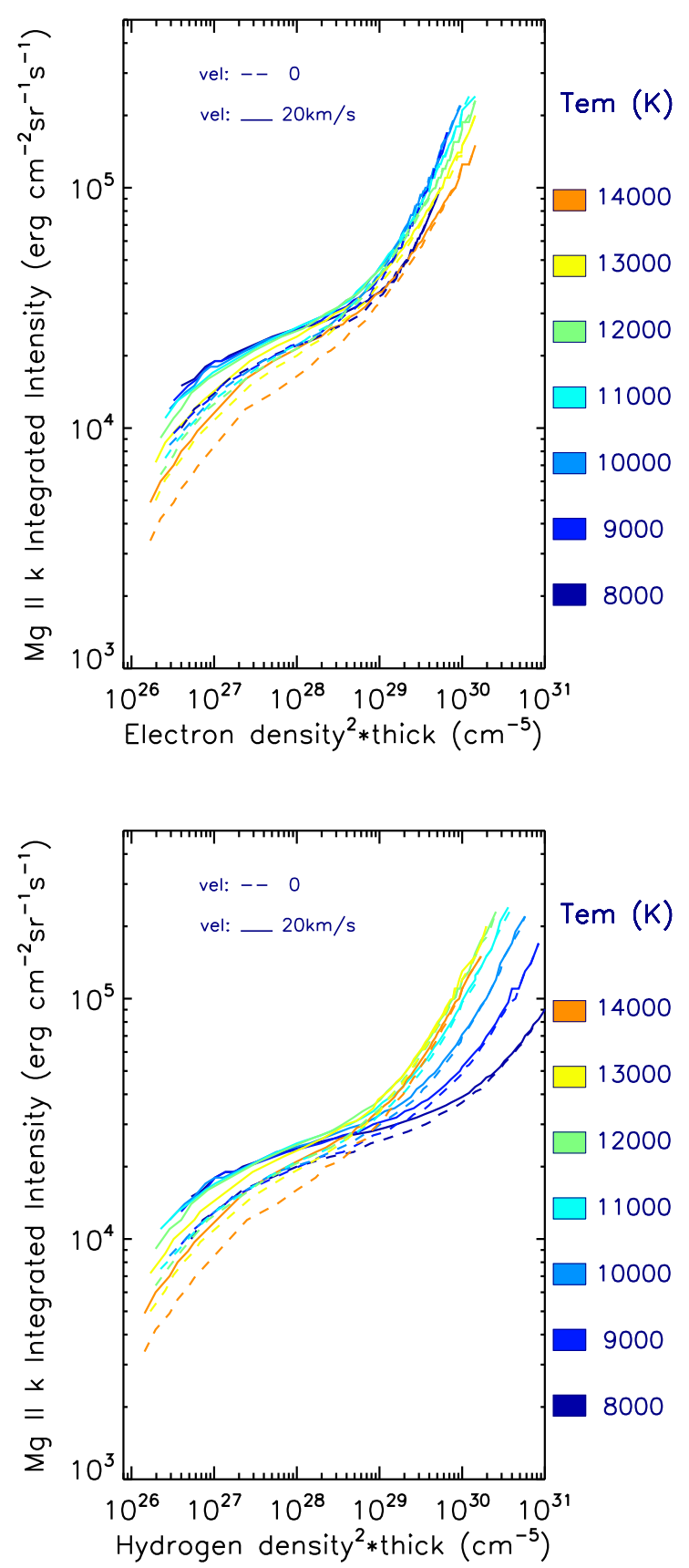

Fig. 5. Integrated $\mathrm{Mg}$ II $\mathrm{k}$ line intensity as a function of the electron $\mathrm{EM}$ (top panel) and the hydrogen EM (bottom panel), for 0 (dashed lines) and $20 \mathrm{~km} \mathrm{~s}^{-1}$ (plain lines) radial velocity, for a thickness of $1000 \mathrm{~km}$.

hydrogen or electron EM on the other hand (bottom panel of Fig. 7). This is no surprise since the EM is proportional to the (effective) thickness.

This means that if observers know the altitude of the prominence plasma blob and the underlying chromosphere (easily obtained in the Mg II $\mathrm{k}$ and $\mathrm{h}$ lines with an IRIS mosaic), if they can measure the plane of sky (POS) velocity, if they assume typical values of microturbulence and temperature, and if they derive the thickness of the blob from its POS extent, they can rely upon a unique relationship between the measured $I_{\mathrm{k}}\left(\right.$ or $\left.I_{\mathrm{h}}\right)$ and the hydrogen and electron EMs. From their guess on the thickness, they can compute the square of the hydrogen and electron densities. Consequently, the derivation of the densities is then rather accurate if one assumes a filling factor equal to one. If one also has access to 


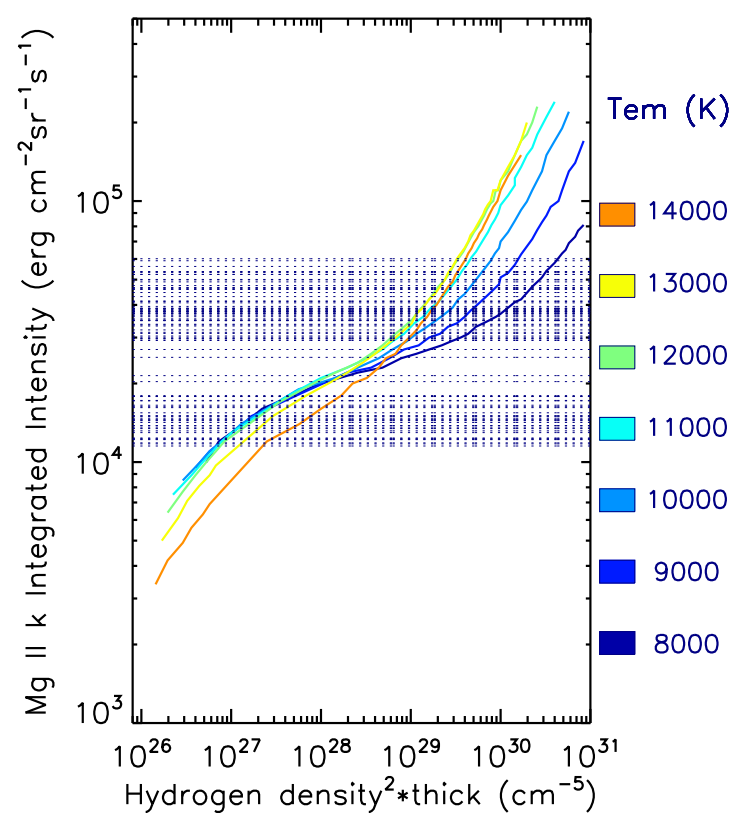

Fig. 6. Integrated $\mathrm{Mg}_{\text {II }} \mathrm{k}$ line intensity as a function of the hydrogen EM for a zero radial velocity, with observed intensities from Paper I superimposed (horizontal dotted lines).

a local value of the density (through, e.g., a ratio of spectral lines sensitive to density), one can derive the filling factor.

Actually, one can also proceed in the reverse way, as did Heinzel et al. (1996). They obtained the electron density from polarimetric measurements in $\mathrm{H} \alpha$ and $\mathrm{He}$ I D3 lines and from the theoretical correlation of the EM with the $\mathrm{H} \alpha$ integrated intensity (GHV), then they derived a large range of "effective" thicknesses. They also concluded that the total column mass in the direction perpendicular to the prominence sheet is fairly constant.

\section{Conclusion}

Our Mg II 1D modeling has been performed with the aim of deriving prominence plasma properties through the pixel-bypixel spectro-imaging presently performed by IRIS. We used the most accurate computation of the $\mathrm{k}$ and $\mathrm{h}$ profiles incident on the prominence, where, depending on the altitude of the prominence, these profiles derive from the point-by-point emission of all areas within the horizon of the prominence. Of course, this can be directly applied to the mosaic images preceding or/and following any IRIS observation (see Paper I). The computation presented here takes into account typical quiet Sun conditions and consequently applies to quiescent prominences only. Although we computed the full profiles of the $\mathrm{Mg}$ II lines involved (including subordinate ones), we focused here on the integrated $\mathrm{k}$ and $\mathrm{h}$ intensities as was done with the hydrogen lines by HGV.

Unsurprisingly, the $\mathrm{Mg}_{\mathrm{II}} \mathrm{k}$ and $\mathrm{h}$ intensities as a function of EM have the same behavior, so we focused on k only. We established that the $\mathrm{k}$ intensity increases with the hydrogen and electron densities. The correlation does not change much when one includes a radial velocity of $20 \mathrm{~km} \mathrm{~s}^{-1}$ (since we limit ourselves to quiescent prominences), but we must recall that this is only valid for low velocities. Because of the Doppler dimming phenomenon, velocities of the order of $300 \mathrm{~km} \mathrm{~s}^{-1}$ can change the emitted radiation by a factor of five (see Heinzel 2015).

The same behavior takes place between the k intensity and the EM (defined as the integral of the product of the square of
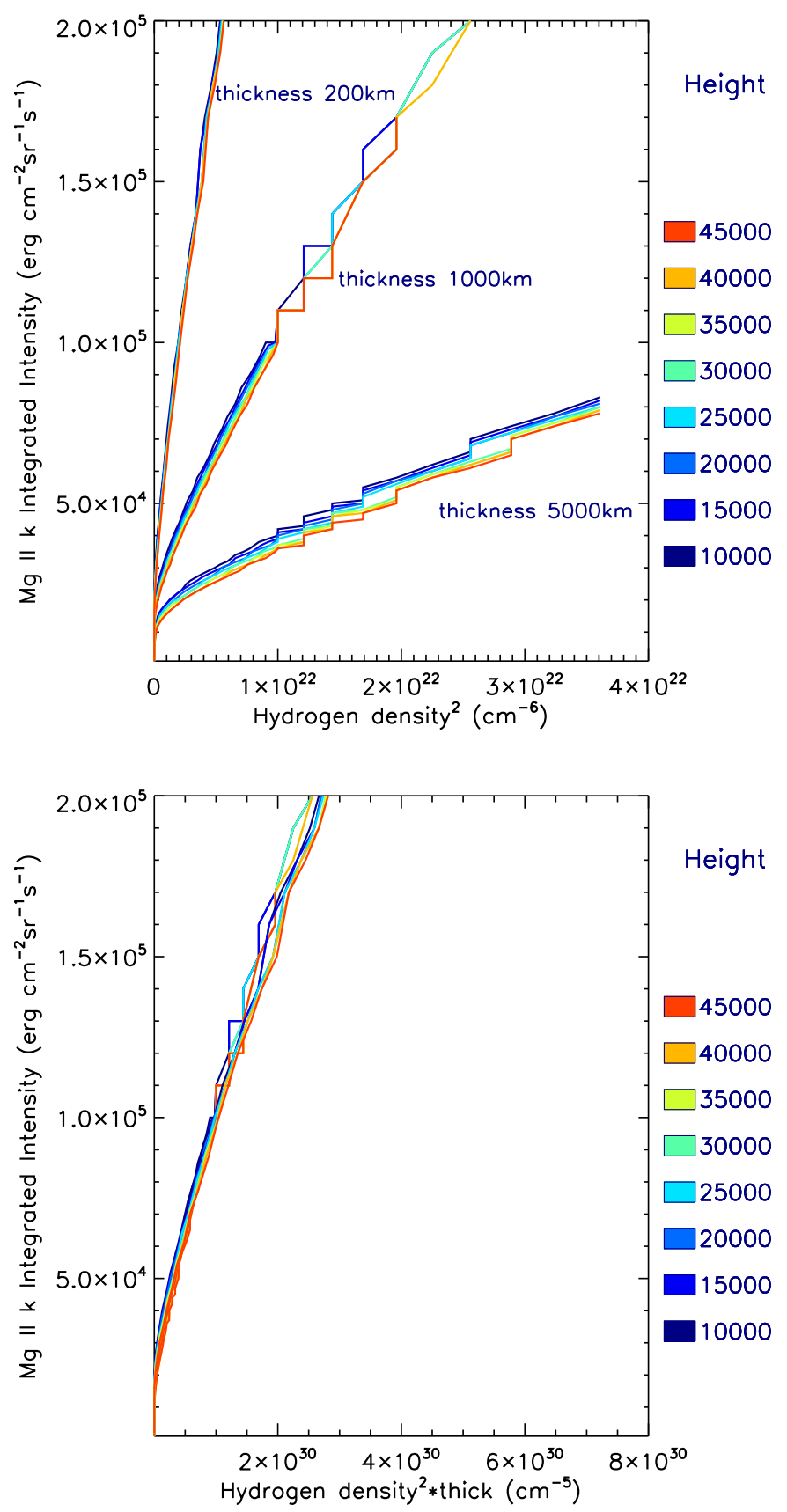

Fig. 7. Integrated $\mathrm{Mg}_{\text {II }} \mathrm{k}$ line intensity as a function of the square of the hydrogen density for three values of the thickness $(200,1000$, and $5000 \mathrm{~km}$, top panel) and as a function of the hydrogen EM (bottom panel) for a thickness of $1000 \mathrm{~km}$. The temperature is $11000 \mathrm{~K}$.

the density with the thickness of the layer). This is valid for both electron and hydrogen densities. Such a correlation is relatively insensitive to temperature if not too high, in such a way that if one is able to determine the (apparent) geometrical thickness, one can derive the density with a good accuracy.

As a conclusion, we think that the correlation found for $\mathrm{Mg}$ II $\mathrm{k}$ (and $\mathrm{h}$ ) intensity is a very useful complement to a similar correlation found for the $\mathrm{H} \alpha$ line of hydrogen (HGV). Although the modeling can be improved in many aspects (e.g., with 2D models or the addition of a PCTR), our results should be useful to prominence observers and analyzers working presently with IRIS, and in the future with the planned Chromospheric LymanAlpha Spectro-Polarimeter (CLASP) 2 experiment. 
Acknowledgements. We thank Dr. Petr Heinzel for his useful comments.

\section{References}

Alissandrakis, C. E., Vial, J.-C., Koukras, A., Buchlin, E., \& Chane-Yook, M. 2018, Sol. Phys., 293, 20

Brown, G. M., \& Labrosse, N. 2018, Sol. Phys., 293, 35

De Pontieu, B., Title, A. M., Lemen, J. R., et al. 2014, Sol. Phys., 289, 2733

Gontikakis, C., Vial, J.-C., \& Gouttebroze, P. 1997, A\&A, 325, 803

Gouttebroze, P., Heinzel, P., \& Vial, J. C. 1993, A\&AS, 99, 513

Gunár, S., Heinzel, P., Schmieder, B., Schwartz, P., \& Anzer, U. 2007, A\&A, 472,929

Heasley, J. N., \& Mihalas, D. 1976, ApJ, 205, 273

Heasley, J. N., \& Milkey, R. W. 1976, ApJ, 210, 827

Heasley, J. N., \& Milkey, R. W. 1983, ApJ, 268, 398

Heinzel, P. 2015, in Solar Prominences, eds. J. C. Vial, \& O. Engvold, Astrophys. Space Sci. Lib., 415, 103

Heinzel, P., \& Anzer, U. 2001, A\&A, 375, 1082

Heinzel, P., Gouttebroze, P., \& Vial, J.-C. 1994, A\&A, 292, 656
Heinzel, P., Bommier, V., \& Vial, J. C. 1996, Sol. Phys., 164, 211

Heinzel, P., Anzer, U., \& Gunár, S. 2005, A\&A, 442, 331

Heinzel, P., Vial, J.-C., \& Anzer, U. 2014, A\&A, 564, A132

Jejčič, S., Schwartz, P., Heinzel, P., Zapiór, M., \& Gunár, S. 2018, A\&A, 618, A88

Labrosse, N. 2015, in Solar Prominences, eds. J. C. Vial, \& O. Engvold, Astrophys. Space Sci. Lib., 415, 131

Labrosse, N., Heinzel, P., Vial, J., et al. 2010, Space Sci. Rev., 151, 243

Paletou, F., Vial, J.-C., \& Auer, L. H. 1993, A\&A, 274, 571

Parenti, S. 2015, in Solar Prominences, eds. J. C. Vial, \& O. Engvold, Astrophys. Space Sci. Lib., 415, 61

Sahal-Brechot, S., Malinovsky, M., \& Bommier, V. 1986, A\&A, 168, 284

Schmit, D., Bryans, P., De Pontieu, B., et al. 2015, ApJ, 811, 127

Vial, J. C. 1982, ApJ, 254, 780

Vial, J. C., \& Engvold, O. 2015, in Solar Prominences, Astrophys. Space Sci. Lib., 415

Vial, J.-C., Pelouze, G., Heinzel, P., Kleint, L., \& Anzer, U. 2016, Sol. Phys., 291, 67

Zhang, P., Buchlin, E., \& Vial, J. C. 2019, A\&A, accepted 